\title{
Robust Transfer Function Identification via an Enhanced Magnitude Vector Fitting Algorithm
}

\author{
Luciano De Tommasi ${ }^{1}$, Bjørn Gustavsen ${ }^{2}$ and Tom Dhaene ${ }^{3}$ \\ ${ }^{1}$ Energy Research Center of the Netherlands - NL-1755 ZG Petten, The Netherlands \\ ${ }^{3}$ SINTEF Energy Research - N-7465 Trondheim, Norway \\ ${ }^{4}$ Ghent University - IBBT, Depart. of Information Technology (INTEC) - Gaston Crommenlaan 8, B-9050 \\ Ghent, Belgium
}

\section{ABSTRACT}

The paper introduces an enhanced version of the magnitude Vector Fitting (magVF) algorithm, a robust procedure for the identification of a transfer function from magnitude frequency domain data. The approach is based on the rational approximation of the magnitude square function with enforcement of symmetric poles and zeros, followed by the elimination of poles and zeros located in the right half plane. The obtained transfer function is stable and of minimum phase shift type. Robustness and accuracy of the basic magVF algorithm are enhanced by enforcing that the magnitude square rational function is non-negative definite and by introducing a new method to remove purely imaginary conjugate poles from the approximation. Practical application of the proposed approach is demonstrated for measured transformer responses and transmission line propagation functions.

\section{Keywords: Rational approximation, magnitude data, vector fitting, minimum phase shift function.}

\section{INTRODUCTION}

Rational fitting from frequency domain responses is commonly applied to the modeling of devices and systems for use in electromagnetic transient studies. In some situations it is desirable to model the behavior from only the magnitude spectrum. For instance, in transmission line modeling by the traveling wave method, the propagation function has traditionally been fitted by a minimum-phase rational function starting from the magnitude data [1]. In transformer condition monitoring, the parametrization of frequency sweep measurements (FRA) by rational fitting [2] is difficult to apply to some cases of past measurements where only the magnitude data is available. Other applications involving magnitude data are found in [3] (radar) and [4] (high-power microwave). A review of the models used in such applications is however out of the scope of the present work. 
This paper describes a robust procedure to identify a complex transfer function of a linear timeinvariant (LTI), causal and stable system, based on a set of samples of its magnitude frequency response. The approach consists of building a rational approximation of the magnitude square response, followed by spectral factorization of the identified rational function. The latter step consists in discarding poles and zeros located in the right half plane. The complete procedure enables to determine a minimum-phase rational function whose magnitude approximates the given magnitude response. The basic idea of this approach was presented earlier (e.g. [5]) but its accuracy and robustness was limited due to the approximation methods available at that time. In the meantime much progress has been made in this field and this can be successfully exploited to obtain very accurate high order approximations.

The Vector Fitting (VF) algorithm [6-7] has already been introduced in [8] to identify high frequency models of potential transformers from magnitude data. However, complicated magnitude responses demand a more robust implementation in order to achieve accurate approximations. In [9], the magnitude Vector Fitting (magVF) approach was introduced and applied to approximation of the propagation function of an overhead transmission line. It can enforce the proper form of the rational function via symmetrical basis functions, so allowing an accurate magnitude fitting via spectral factorization. Afterward, this approach was successfully applied to modeling of interconnects from S-parameters [10]. Moreover, the magVF symmetrical basis functions have been successfully exploited to model frequency responses of microwave components parametrized with respect to some geometrical parameters [11]. In such case, they are suitable as basis function describing the variations given by geometrical parameters on the component frequency response.

The original work described in this paper was motivated by the fact that the basic magVF algorithm presented in [10] may fail when applied to the identification of accurate, high order models of measured frequency responses. Such accuracy limitations are overcome by the enhanced magVF version through the combination of two novel ideas, namely: 1) the enforcement of the non-negative definiteness of the magnitude square rational approximation via a constrained identification based on convex optimization; 2) an improved approach to remove purely imaginary pairs of poles from the magnitude square rational approximation. 
Numerical results show that these improvements enable the correct identification of high order models of measured frequency responses.

The paper is organized as follows. Section 2 reviews the basic idea of magnitude fitting via spectral factorization. Section 3 reviews the rational approximation of a magnitude square function by means of a VF formulation exploiting the symmetric basis functions. In order to highlight the differences with the standard VF approach [6], it is first shown the application of VF to a magnitude square function (subsection 3.1) as proposed in [8], then the same approximation problem is tackled by assuming symmetric basis functions (subsection 3.2) [10]. Sections 4 and 5 include both the novel features of the enhanced magVF algorithm. The magnitude square of a transfer function of a physically realizable system must not have any singularities and must be non-negative definite. The first condition (Section 4) can be achieved by removing the pairs of imaginary poles which may appear in the magnitude square approximation. Imaginary poles can be either transformed into their real counterparts (as proposed in [10]) or into two symmetrical pairs of complex conjugate poles (i.e. the new approach introduced in this paper). The second condition (Section 5) is enforced by means of a constrained identification approach based on convex optimization. Section 6 reviews the importance of symmetrical basis functions by showing an example of rational approximation where the VF [6] fails, whereas the magVF approach [10] does work. Section 7 shows that the enhanced magVF algorithm converges to accurate results even in the case of approximation of complicated measured frequency responses (transformer modeling problem), whereas the basic magVF [10] is not accurate. In particular, we show that: 1) the magVF approximation may be asymptotically inaccurate when the non-negative definiteness of the magnitude square function is not enforced by means of the constrained identification approach introduced in section $5 ; 2$ ) transformation of purely imaginary poles into real ones may result in a severe loss of accuracy; on the other hand transformation into complex conjugate pairs (as described in section 4) correctly solves the approximation problem.

Section 8 illustrates the application of magVF to transmission line modeling [9]. Finally, section 9 concludes the paper. 


\section{Magnitude FitTing Via SPECTRAL FACtORIZATION}

The Laplace transform $F s$ of the impulse response $f t$ of a causal and stable LTI system is uniquely determined by the value of phase angle of $F \quad 0$ and the function:

$$
F \quad j \omega^{2}=F \quad j \omega \quad F^{i} j \omega
$$

provided that $F j \omega$ satisfies the Paley-Wiener condition [12]:

$$
\int_{-\infty}^{\infty} \frac{\ln F j \omega}{1+\omega^{2}} d \omega<+\infty .
$$

In the followings, we assume that $F$ is a rational function:

$$
F s=F_{0} \frac{\prod_{m=1}^{M} s-z_{m}}{\prod_{n=1}^{N} s-p_{n}}
$$

where $M=N-1$ or $M=N, \quad F_{0}$ is a positive real constant and both poles $\left\{p_{n}\right\}_{n=1 . . N}$ and zeros $\left\{z_{m}\right\}_{m=1 . . M}$ are either real or occur in complex conjugate pairs. The magnitude square function (1) becomes:

$$
F j \omega{ }^{2}=F_{0}^{2} \frac{\prod_{m=1}^{M}\left[j \omega-z_{m}-j \omega-z_{m}\right]}{\prod_{n=1}^{N}\left[j \omega-p_{n}-j \omega-p_{n}\right]},
$$

which can be written as:

$$
\begin{aligned}
& F j \omega^{2}=F_{0}^{2} \frac{\prod_{m=1}^{M}\left[\begin{array}{ll}
j \omega-z_{m} & j \omega+z_{m}
\end{array}\right]}{N} \text {. }
\end{aligned}
$$

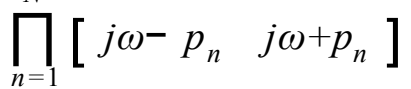

This equation suggests to approximate the magnitude square of a tabulated function $\left\{F s_{k}=j \omega_{k}{ }^{2}\right\}_{k=1 . . K}$ by including both poles/zeros belonging to the left hand side of the complex plane and their symmetrical counterparts in the right hand side (6). Afterward, the spectral factorization can 
be applied: right hand poles and zeros are discarded in order to find the corresponding minimum-phaseshift transfer function, whose magnitude approximates $F s$.

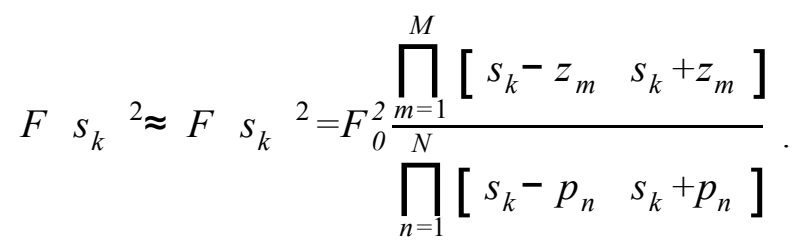

Assuming that $\operatorname{Re}\left\{z_{m}\right\} 0$ for $m=1 \ldots M$ and $\operatorname{Re}\left\{p_{n}\right\} 0$ for $n=1 \ldots N$, we get:

$$
F s_{k}=F_{0} \frac{\prod_{m=1}^{M} s_{k}-z_{m}}{\prod_{n=1}^{N} s_{k}-p_{n}} \approx F s_{k} .
$$

The pole-zero form (6) corresponds to the following symmetrical form (8), where $r_{0}=0$ when $M=N-1$ and $r_{0}=F_{0}^{2}$ when $M=N$ :

$$
F s_{k}^{2}=r_{0} \sum_{n=1}^{N} \frac{r_{n}}{s_{k}-p_{n}}-\frac{r_{n}}{s_{k}+p_{n}}
$$

Note that standard VF algorithm, when applied to $F s_{k}{ }^{2}$ may end up with slightly asymmetrically perturbed poles and residues:

$$
F^{\prime} s_{k}=r_{0} \sum_{n=1}^{N} \frac{r_{n}}{s_{k}-p_{n}}-\frac{r_{n} \Delta r_{n}}{s_{k} p_{n} \Delta p_{n}} \approx F s_{k}^{2}
$$

where $F^{\prime}$ is not necessarily real and non-negative definite (therefore the magnitude square notation $F s_{k}{ }^{2}$ cannot be used).

If the approximation (9) is fairly accurate then the perturbations $\Delta r_{n}$ and $\Delta p_{n}$ are necessarily small, since when $\Delta r_{n} \neq 0$ and $\Delta p_{n} \neq 0$, then $F^{\prime} s_{k}$ has an imaginary part (whereas $F s^{2}$ is purely real). Nevertheless, rational functions like (9), after the spectral factorization (6-7), give inaccurate approximations of $F s \quad$. Therefore, in the next section we will review the magnitude Vector Fitting 
algorithm, which enforce the pole/zero symmetry by using a proper set of basis functions, so giving $\Delta r_{n}=0$ and $\Delta p_{n}=0$ in (9).

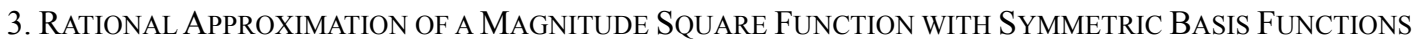

\subsection{Vector Fitting Applied to Magnitude Square Data}

The application of the standard VF algorithm to a magnitude square function [8] leads to the following linear least squares (LS) problem (pole identification step) [6]:

$$
F^{2} \sigma_{\text {rat }} s-F^{2} \sigma_{\text {rat }} s \approx 0 .
$$

Equation (10) represents the $K$ equations obtained for different values of $s_{k}=j \omega_{k}$, where $k=1 \ldots K$, and

$$
\begin{gathered}
F^{2} \sigma_{\text {rat }} s=\sum_{n=1}^{2 \mathrm{~N}} \frac{c_{n}}{s-a_{n}{ }^{0}}+d, \\
\sigma_{\text {rat }} s=\sum_{n=1}^{2 \mathrm{~N}} \frac{c_{n}}{s-a_{n}^{0}} 1 .
\end{gathered}
$$

The unknowns of (10) are $\left\{c_{n}\right\}, d,\left\{\bar{c}_{n}\right\}$, whereas $\left\{a_{n}^{0}\right\}$ is an initial guess of poles. Once (10) is solved, an improved pole set $\left\{a_{n}{ }^{1}\right\}$ for $F^{2}$ is given by the zeros of $\sigma_{r a t} s$. In fact, equation (10) shows that:

$$
F^{2} \approx \frac{F^{2} \sigma_{r a t}}{\sigma_{r a t}}
$$

This process is known as pole relocation. In the second step, the known poles $\left\{a_{n}{ }^{1}\right\}$ are used to solve the following LS problem (residue identification step):

$$
F^{\prime}=r_{0} \sum_{n=1}^{2 \mathrm{~N}} \frac{r_{n}}{s-a_{n}{ }^{1}} \approx F^{2},
$$

with unknowns $\left\{r_{n}\right\}$. The solution of (13) eventually completes the rational approximation $F^{2}$. The whole procedure may be iterated by replacing the initial guess $\left\{a_{n}{ }^{0}\right\}$ with the improved pole set $\left\{a_{n}{ }^{1}\right\}$. 
Convergence occurs in few iterations [6].

\subsection{Symmetric Basis Functions}

In order to enforce that poles of $F^{\prime}$ occur in opposite pairs, a magnitude Vector Fitting (magVF) algorithm has been introduced [9-10]. The pole identification scheme of (10) and (12) still holds, but now using the expansions (14) instead of (11):

$$
\begin{gathered}
F^{2} \sigma_{r a t}=\sum_{n=1}^{N} c_{n} \frac{1}{s-a_{n}^{0}}-\frac{1}{s+a_{n}^{0}}+d, \\
\sigma_{r a t}=\sum_{n=1}^{N} \bar{c}_{n} \frac{1}{s-a_{n}^{0}}-\frac{1}{s+a_{n}^{0}} \quad 1
\end{gathered}
$$

The residues identification step is replaced by:

$$
F^{\prime}=r_{0} \sum_{n=1}^{N} r_{n} \frac{1}{s-a_{n}{ }^{1}}-\frac{1}{s+a_{n}{ }^{l}} \approx F^{2} \text {. }
$$

In order to compute the relocated pole set $\left\{a_{n}{ }^{1}\right\}$, we express (14b) into pole-zero form:

$$
\sigma_{r a t} s=\sum_{n=1}^{N} \frac{2 c_{n} a_{n}}{s^{2}-a_{n}^{2}} \quad 1=\frac{\prod_{n=1}^{N} s^{2}-\lambda_{n}}{\prod_{n=1}^{N} s^{2}-a_{n}^{2}}
$$

Note that zeros of $\sigma_{r a t} S$ occur in truly opposite pairs. Once that $\sigma_{r a t} s$ is written as function of $s^{2}$, the computation of $\left\{\lambda_{n}\right\}$ requires the solution of the same eigenvalue problem solved with the standard VF algorithm [6]:

$$
\left\{\lambda_{n}\right\}=e i g\left\{A-b c^{T}\right\}
$$

where $A$ is a $N \times N$ real, block diagonal, matrix, $b$ and $c^{T}$ are, respectively, a column and a row real vector, defined as follows: if $a_{n}^{2}$ is real, then the $n^{\text {th }}$ block of $A$ is $a_{n}^{2}, b_{n}=1$ and $c_{n}=2 c_{n} a_{n}$; else if $a_{n}^{2}=a_{n}^{\prime}+j a_{n}^{\prime \prime}$ and $a_{n+1}^{2}=a_{n}^{\prime}-j a_{n}^{\prime \prime}$, then the $n^{\text {th }}$ block of $A$ is the $2 \times 2$ block: 


$$
A_{n}=\left[\begin{array}{cc}
a_{n}^{\prime} & a_{n}^{\prime \prime} \\
-a_{n}^{\prime \prime} & a_{n}^{\prime}
\end{array}\right]
$$

and $b_{n}=2, b_{n+1}=0, c_{n}=\operatorname{Re}\left\{2 \bar{c}_{n} a_{n}\right\}, c_{n+1}=\operatorname{Im}\left\{2 \bar{c}_{n} a_{n}\right\}$.

It is remarked that, since the matrix $A-b c^{T}$ is real, the eigenvalues $\left\{\lambda_{n}\right\}$ either are real or occur in complex conjugate pairs. Hence, eigenvalues can only be real or complex conjugate. The relocated poles are computed by extracting the square root of corresponding eigenvalues:

$$
a_{n}^{1}= \pm \bar{\lambda}_{n}
$$

It is straightforward to verify that each positive real eigenvalue leads to a pair of symmetric real poles, and that each pair of complex conjugate eigenvalues leads to two symmetrical pairs of complex conjugate poles

of $F^{\prime}$. Furthermore, note that a real and negative eigenvalue $\lambda_{n} \quad 0$ corresponds to a pair of imaginary conjugate poles, which cannot be retained in the magnitude square approximation, because it is unphysical. Therefore negative real eigenvalues must be transformed into real or complex conjugate, as discussed in the following section.

Discussion above demonstrated that the magVF can relocate the symmetrical initial pole guess into an improved set of poles, which is still symmetrical; this result holding for both real and complex conjugate poles, i.e. $\Delta p_{n}=0$ in (9). Symmetry of the residues (which implies zero symmetry) is obtained by formulating the residue identification step as in (15), i.e. $\Delta r_{n}=0$ in (9).

In this section the magVF algorithm has been described referring to the standard VF implementation [6]. A 'relaxed' version of magVF is straightforward to obtain from [13].

\section{AVOIDING IMAGINARY POLES IN POLE RELOCATION}

In the previous section, it has been shown that the main difference between magVF and VF is that the improved (relocated) pole set is not directly given by the eigenvalues $\left\{\lambda_{n}\right\}$, but is obtained by extracting the square root.

With the VF algorithm, a positive real eigenvalue represents an unstable poles, which cannot be retained in the rational approximation. Differently, with the magVF algorithm, a negative real eigenvalue 
$\lambda_{n} \quad 0$ corresponds to a pair of purely imaginary poles of the magnitude square rational approximation which have to be discarded as well. In fact, if these poles were used in the residue identification (15), the magnitude square approximation $F^{\prime}$ would eventually contain the (unphysical) pair of fractions:

$$
\frac{j \beta}{s^{-} j \alpha}-\frac{j \beta}{s+j \alpha}
$$

whose magnitude diverges when $s \quad j \alpha$.

The VF algorithm removes the unstable poles by inverting the sign of their real part. This introduces an error which is negligible. A similar approach was also implemented in the magVF formulation [10] to remove negative real eigenvalues. Changing the sign of a $\lambda_{n} \quad 0$ before extracting the square root is equivalent to change the pair of fractions (19) into the pair:

$$
\frac{\beta^{\prime}}{s^{-\alpha}}-\frac{\beta^{\prime}}{s+\alpha} \text {. }
$$

However, it is straightforward to understand that (19) can approximate a resonance peak, whereas (20) cannot. Therefore, differently on the VF, the introduced perturbation can be quite large. Note that the lost contribution of (19) may be compensated in subsequent pole identification iterations by the relocation of complex conjugate pairs of poles. When this happen, negative eigenvalues disappear from (16) and therefore transformation $(19) \rightarrow(20)$ does not apply anymore, so leading to an accurate final approximation. However, we will give in section 7 an example where this does not happen. Hence, an improved method to treat the negative real eigenvalues is needed. The idea behind the new approach is that a more accurate approximation of the pair (19) than (20) will lead to a more accurate approximation when solving the residue identification problem (15). To this end, each conjugate pair of imaginary poles (19) can be changed into two pairs of complex conjugate poles by introducing a small real part:

$$
\frac{\varepsilon_{\beta} j \beta^{\prime \prime}}{s^{-} \varepsilon_{\alpha} j \alpha} \frac{\varepsilon_{\beta}-j \beta^{\prime \prime}}{s^{-} \varepsilon_{\alpha}-j \alpha}-\frac{\varepsilon_{\beta}-j \beta^{\prime \prime}}{s^{-}-\varepsilon_{\alpha} j \alpha}-\frac{\varepsilon_{\beta} j \beta^{\prime \prime}}{s^{-}-\varepsilon_{\alpha}^{-j \alpha}} .
$$

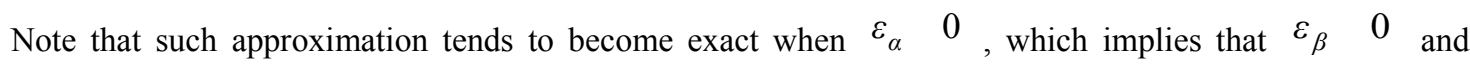
$\beta^{\prime \prime} \frac{\beta}{2}$. Inspired by the recommended procedure for selection of starting poles in VF, we use $\varepsilon_{\alpha}=\alpha / 100$. This is known to avoid ill-conditioning of system of equations [6]. 
Since this approach replaces two fractions with four fractions, the degree of the rational approximation increases during the iterations. This accelerates the convergence.

The magVF iterations start with the transformation $(19) \rightarrow(20)$. Transformation $(19) \rightarrow(21)$ is used only when some $\left\{\begin{array}{ll}\lambda_{n} & 0\end{array}\right\}$ remain in the (16) after a predefined number of pole identification iterations.

\section{ENSURING A NON-NEGATIVE DEFINITE MAGNITUDE SQUARE FUNCTION}

The rational approximation $F^{\prime}$ of the magnitude square function $F^{2}$ must be non-negative definite. This cannot be guaranteed when the unconstrained least squares fitting [6],[10] is applied to pole and residue identification steps. In particular, it may happen that $F^{\prime} s_{k} \quad 0$ for some frequency samples

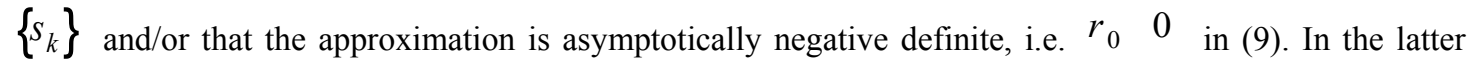
case, it can be corrected by inverting the sign of $r_{0}$, of course worsening the accuracy (an example is given in Section 7).

The non-negative definiteness of the magnitude square approximation can be enforced by means of constrained residue identification. In such case, the following constraints are added to the least square problem (15):

$$
\begin{gathered}
F^{\prime} s_{k} \geq 0, k=1 \ldots K, \\
r_{0} \geq 0 .
\end{gathered}
$$

The least squares problem (15) under the constraints (22) is a constrained norm-2 minimization problem, which can be effectively solved by available convex programming software. The implementation proposed in this paper is based on the public domain software cvx, a package for specifying and solving disciplined convex programs [14-15]. Disciplined convex programs (DCPs) are convex optimization problems which can be described using a limited set of construction rules, known as the DCP ruleset. Problems adhering to the DCP ruleset are automatically verified as convex and converted to a solvable form by the modeling software.

Combining some DCP rules, it can be found that a minimization problem consisting of a convex objective function and "greater than" inequality constraints, where the left side is concave and the right side is convex, is a DCP problem. Problem (15) under the constraints (22) belongs to such category of DCPs. In 
fact, the objective function to be minimized when solving (15) is convex, because attainable by composition of an affine inner component and a convex outer component, i.e. the norm-2 function (affine composition rule). Moreover, left hand sides of (22) are affine (and therefore concave) whereas right hand sides are constant (and therefore convex).

\section{TEST CASE: FITTING OF A RATIONAL FUNCTION}

In this section the application of basic magVF approach [10] is reviewed by showing a first test case. We consider the $18^{\text {th }}$ order rational function $G S$, whose poles and residues are given in Table 1 .

$$
G s=\sum_{n=1}^{N} \frac{c_{n}}{s-a_{n}}
$$

In order to compare the VF and magVF algorithms, 200 frequency samples $\left\{G j \omega_{k}\right\}$ have been evaluated, with $\left\{\omega_{k}\right\}$ linearly spanning the interval (1 Hz, $\left.20 \mathrm{kHz}\right)$.

Fig. 1 shows that both VF and magVF accurately fit the magnitude of the original function using 36 poles (ten iterations). Fig. 2 shows that the phase angles of VF and magVF approximations do not match the reference: this happens because the spectral factorisation constrains the identified functions to be minimum phase shift, whereas $G$ is not.

Constrained identification (Section 5) was not used in this example.

As a second test case, we consider the non-rational function:

$$
G^{\prime} \quad s=\begin{array}{llll} 
& s & +n & s
\end{array}
$$

obtained by adding Gaussian noise $n s \quad$ with mean $\mu=0$ and standard deviation $\sigma=0.01$ to the magnitude of $G s$

This example aims to reproduce the situation where the magnitude function is obtained via direct measurements of a real system. In order to compare the accuracy of VF and magVF, we consider the (absolute) rms-error:

$$
\varepsilon=\overline{\sum_{k=1}^{K} G^{\prime} j \omega_{k}-G_{r a t}^{\prime} j \omega_{k}{ }^{2}}
$$


where $G_{r a t}^{\prime} S$ is the rational approximation (obtained with VF or magVF).

Several simulations with different noise realizations were done. VF was mostly found inaccurate (see also [10]). On the other hand, magVF turned out to be more reliable and showed a fast convergence. An example of this is shown in Fig. 3. The failure with VF occurs when the spectral factorization is applied to (6) in order to get (7). In fact, in order to get an accurate magnitude approximation, spectral factorization requires the proper form of the magnitude square rational function, given by the magVF method (i.e. $\Delta r_{n}=0$ and $\Delta p_{n}=0$ in (9)). It is observed in Fig. 5 that the large errors by VF remain in subsequent iterations.

\section{APPLICATION TO TRANSFORMER MODELING}

In this example we consider a 2 MVA 3-winding rectifier transformer. The admittance matrix was measured with respect to only two windings, with the terminals of the third winding $(3 \mathrm{kV})$ open [16]. The neutral of the $2 \mathrm{kV}$ winding was grounded during the measurements. The $6 \mathrm{x} 6$ admittance matrix $\mathbf{Y}$ was measured with respect to the $10 \mathrm{kV}$ and $2 \mathrm{kV}$ winding, giving 401 linearly spaced samples between $1 \mathrm{kHz}$ and $1 \mathrm{MHz}$.

The application of the proposed magVF algorithm is demonstrated for admittance elements $y_{11} S$ and $y_{12} S$.

The admittance $y_{11} s$ was fitted using 48 poles and five iterations, with inverse magnitude leastsquares weighting. Any imaginary poles were converted in to real poles by the transformation (19) $\rightarrow(20)$. Moreover, both unconstrained and constrained residue identifications (22) were applied. Fig. 5 shows that unconstrained residue identification results in an approximation with incorrect asymptotic behavior. This is due to the constant term $r_{0}$ being negative. On the other hand, a perfect agreement is achieved with the constrained identification. Fig. 6 shows the phase angles: in this case the agreement is satisfactory, since both the reference and the magVF approximation are minimum phase shift functions. They are however not perfect, because the phase angle information is not exploited in the fitting process, and because of measurement errors 
The admittance $y_{12} S$ is fitted again using inverse weighting. Suppression of imaginary poles is now achieved by means of transformation into complex conjugate poles i.e. (19) $\rightarrow(21)$, since the transformation into real poles does not suffice to remove them, see Fig. 7 . The fitting process starts with 40 poles, and after four iterations ends up with 76 (each pair of imaginary poles is replaced with two pairs of complex conjugate poles). Fig. 8 shows the phase angles: in this case a partial agreement cannot be achieved, since the reference function is not a minimum phase shift function.

\section{ApPliCATION to TRANSMisSiOn Line ModELING}

In this section we show the application of the proposed magnitude fitting algorithm to transmission line modeling.

Frequency dependent transmission line models used in the simulation of power system transients are usually formulated via the method of characteristics (MoC), due to its high computational efficiency [1722]. Such methods demand the rational approximation of the characteristic admittance $Y_{C}$ and the propagation function $H$, so enabling efficient time domain simulations by recursive convolutions [18].

Several approximation techniques of $H$ require to approximate its modes with a rational function plus a single time delay [19-22]:

$$
h_{m} \approx \sum_{n=1}^{N} \frac{r_{n}}{s-a_{n}} e^{-s \tau}
$$

The assumption of a rational function plus a delay term on the right side of (26) enables an accurate fitting of $h_{m}$ with relatively low orders.

The rational function in (26) can be computed either with the VF algorithm (after that a suitable time delay has been compensated) (27) or by means of a magnitude fitting approach (28):

$$
\begin{gathered}
h_{m} e^{s \tau} \approx \sum_{n=1}^{N} \frac{r_{n}}{s-a_{n}}, \\
h_{m} \approx \sum_{n=1}^{N} \frac{r_{n}}{s-a_{n}} .
\end{gathered}
$$

In the latter case, a single delay is associated to the rational approximation and subjected to final optimization in order to minimize the rms-error of the (26): 


$$
\varepsilon=\overline{\sum_{k=1}^{K} h_{m} j \omega_{k}-\sum_{n=1}^{N} \frac{r_{n}}{j \omega_{k}-a_{n}} e^{-j \omega_{k} \tau^{\prime}}} .
$$

The latter approach (28-29) has been implemented in line models [1] in several EMTP-like programs. The approximation (29) is computed via asymptotic magnitude fitting using only real poles and zeros, plus a subsequent refinement (see also [22]). Asymptotic fitting of magnitude functions [1] allocates (real) poles and zeros by tracking the magnitude of the original function as function of frequency. A new pole or zero is allocated whenever the asymptote of the fitting function deviates from the original function by more than a predefined tolerance. Afterward, the obtained poles and/or zeros are further refined using a suitable optimization procedure (e.g. the Gauss-Newton non-linear least squares algorithm). The procedure places all poles and zeros in the left half-plane, thereby giving a rational approximation which is stable and of minimum phase shift.

Although asymptotic magnitude fitting offers robust accurate approximations and is currently used, it often requires high orders, therefore resulting in slow time domain simulations. In the present work, we propose instead to replace the asymptotic magnitude fitting with the magVF algorithm to achieve a lower order fitting.

As example of magVF identification of the modal components of a propagation function matrix, we consider an underground cable system (length $5 \mathrm{~km}$ ), which has six propagation modes. Only four modes are shown since the three coaxial modes are nearly identical at high frequencies.

Fig. 9 shows that the approximation of all the modes of $H$ is achieved with a high accuracy, respectively using 10,10,15 and 20 poles. All the magnitude square approximations are unweighted and unconstrained.

It is worth noting that when the unconstrained magnitude square fitting is applied to modes decaying steeply (typically the ground mode), the rational function may exhibit a negative undershoot, which can be easily eliminated with the simple procedure illustrated in the following, which does not require constrained identification as introduced in Section 5.

For the shown example, the rational approximation of the magnitude square of the ground mode has a pair of conjugate purely imaginary zeros $\pm z_{0}= \pm j 5.9310^{7}$, and therefore crosses the zero line at the 
corresponding frequency $\omega_{0}=\frac{z_{0}}{2 \pi}=9.44 \mathrm{MHz}$. If the imaginary zeros are removed, the negative undershoot is also removed, since the rational function cannot cross the zero line anymore. Hence, we replace the pair of imaginary zeros $\pm z_{0}= \pm j \omega_{0}$ with the real pair $\pm z_{0 \mathrm{r}}= \pm \omega_{0}$. Fig. 10 shows the effect of such correction applied to the ground mode of the given example. The introduced perturbation is seen to be negligible.

The method of magVF can also be applied by the Universal Line Model [19]. This is discussed in Appendix.

\section{CONCLUSION}

Rational modeling from magnitude frequency responses can be achieved by fitting the magnitude square response followed by spectral factorization to recover a minimum phase shift magnitude approximation. In order to improve the robustness of the fitting procedure, a new formulation of the Vector Fitting algorithm was introduced in [9-10]. It uses a set of symmetrical basis functions to enforce that pole/zero occur in opposite pairs.

In this work, an enhanced version of the magVF algorithm has been presented. It includes a robust method to ensure the physicality of the magnitude-square approximation by removing the presence of imaginary poles and enforcement of the magnitude square rational approximation non-negative definiteness via convex optimization.

The magVF approach was demonstrated to be suitable for the modeling of transformers from measured magnitude of Y-parameters and for fitting the modal components of a transmission line propagation function. In the latter case, the accuracy is improved with respect to the traditional asymptotic Bode fitting approach [1].

\section{APPENDIX: UNIVERSAL LINE MODEL}

An accurate procedure for fitting the propagation function $H S$ in the phase domain is provided by the Universal Line Model [19]. According to this scheme, $H S$ is fitted in the phase domain taking the poles $\left\{a_{m n}\right\}$ and delays $\left\{\tau_{m}\right\}$ of the modes as known quantities: 


$$
H \quad s=\sum_{m=1}^{M} \sum_{n=1}^{N} \frac{R_{m n}}{s^{-} a_{m n}} e^{-s \tau_{m}} .
$$

The extraction of poles by mode fitting can now be achieved by magVF, followed by delay extraction. A more accurate result for the mode fitting can in general be obtained by simultaneous optimization of poles and delays [20],[22]. However, that approach is less effective due to the need for calling VF repeatedly. Furthermore, the improved accuracy of (30) by using optimization is often small since the modes are fitted independently.

\section{ACKNOWLEDGMENT}

This work was supported in part by the European Commission through the Marie Curie program under contract number MTKI-CT-2006-042477 (O-MOORE-NICE! Project). The first author executed the work at University of Antwerp and NXP semiconductors.

\section{REFERENCES}

[1] J.R. Marti, "Accurate modelling of frequency dependent transmission lines in electromagnetic transient simulations", IEEE Trans. on Power Apparatus and Systems, Vol. 101, No. 1 (1982), pp. 147-157.

[2] P. Karimifard G.B. Gharehpetian and S. Tenbohlen, "Determination of axial displacement extent based on transformer winding transfer function estimation using vector-fitting method”, European Transactions on Electrical Power, vol. 18, no. 4, pp. 423436, 2008

[3] Jie Yang and T.K. Sarkar, "Interpolation/Extrapolation of Radar Cross-Section (RCS) Data in the Frequency Domain Using the Cauchy Method", IEEE Trans. Antennas and Propagation, vol. 55, no. 10, pp. 2844 - 2851, 2007.

[4] F.M. Tesche, "On the use of the Hilbert transform for processing measured CW data", IEEE Trans. Electromagnetic Compatibility, vol. 34, no. 3, pp. $259-266,1992$.

[5] M.T. Jong and K.S. Shanmugam, "Determination of a transfer function from amplitude frequency response data", Int. J. Control, vol. 25 , no. 6, pp. 941-948, 1977.

[6] B. Gustavsen and A. Semlyen, "Rational approximation of frequency domain responses by vector fitting", IEEE Trans. Power Delivery, vol. 25, no. 3, pp. $1052-1061,1999$.

[7] W. Hendrickx and T. Dhaene, "A Discussion of 'Rational Approximation of Frequency Domain Responses by Vector Fitting',", IEEE Trans. Power Systems, vol. 21, no. 1, pp. 441-443, 2006.

[8] Zhongyuan Zhang, Guishu Liang, Xiang Cui and Maolin Wu, "A high frequency transfer function model of potential transformer in GIS", Proc. 3rd Int. Symp. on Electromagnetic Compatibility, pp. 186-189, 2002. 
[9] L. De Tommasi and B. Gustavsen, "Low Order Transmission Line Modeling by Modal Decomposition and Minimum Phase Shift Fitting”, 10th IEEE Workshop on Signal Propagation on Interconnects, pp. 89-92, 2006.

[10] L. De Tommasi, B. Gustavsen and T. Dhaene, "Accurate Macromodeling Based on Tabulated Magnitude Frequency Responses", 12th IEEE Workshop on Signal Propagation on Interconnects, 2008.

[11] D. Deschrijver, T. Dhaene and D. De Zutter, "Robust Parametric Macromodeling Using Multivariate Orthonormal Vector Fitting”, IEEE Trans. on Microwave Theory and Techniques, Vol. 56, No. 7 (2008), pp. 1661 - 1667.

[12] D.A. Overdijk, van de Wouw and A. De Kraker, "Methods in spectral factorization. A modelling and design tool.", Z. Angew. Math. Mech., vol. 81, no. 2, pp. 140-144, 2001.

[13] B. Gustavsen, "Improving the pole relocating properties of vector fitting", IEEE Trans. Power Delivery, vol. 21, no. 3, pp. 1587 $-1592,2006$.

[14] M. Grant and S. Boyd, "CVX: Matlab software for disciplined convex programming", web page and software: http://stanford.edu/ boyd/cvx, June 2008.

[15] M. Grant and S. Boyd, "Graph implementations for nonsmooth convex programs", to appear in Recent Advances in Learning and Control (a tribute to M. Vidyasagar), V. Blondel, S. Boyd, and H. Kimura, editors. Springer, 2008. http://stanford.edu/ boyd/graph dep.html.

[16] B. Gustavsen, "Application of vector fitting to high frequency transformer modeling”, Proc. Int. Conference on Power Systems Transients, September 28- October 3, New Orleands, USA, pp. 1-6.

[17] G. Miano and A. Maffucci, "Transmission Lines and Lumped Circuits”, Academic Press, 2001.

[18] A. Semlyen and A. Dabuleanu, "Fast and accurate switching transient calculations on transmission lines with ground return", IEEE Trans. on Power Apparatus and Systems, Vol. 94, No. 2 (1975), pp. 561-571.

[19] A. Morched, B. Gustavsen and M. Tartibi, "A universal model for accurate calculation of electromagnetic transient on overhead lines and underground cables", IEEE Trans. on Power Delivery, Vol. 14, No. 3 (1999), pp. 1032-1038.

[20] B. Gustavsen, "Time delay identification for transmission line modeling", Proceedings of 8th IEEE Workshop on Signal Propagation on Interconnects, (2004), pp. 103-106.

[21] B. Gustavsen and A. Semlyen, "Simulation of transmission line transients using vector fitting and modal decomposition", IEEE Trans. on Power Delivery, Vol. 27, No. 2 (1998), pp. 605-614.

[22] L. De Tommasi and B. Gustavsen, "Accurate transmission line modeling through optimal time delay identification", Proceedings of the International Conference on Power Systems Transients, IPST 2007 in Lyon, June 4th-7th, 2007. 


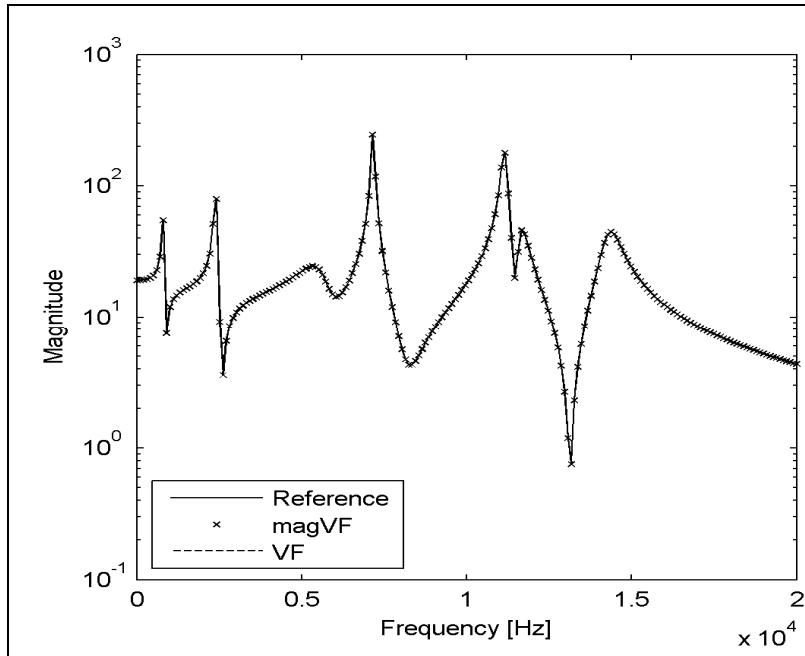

Fig. 1 Magnitude of the reference function $G$ (solid line) compared to magVF approximation (dashed line) and VF approximation (cross).

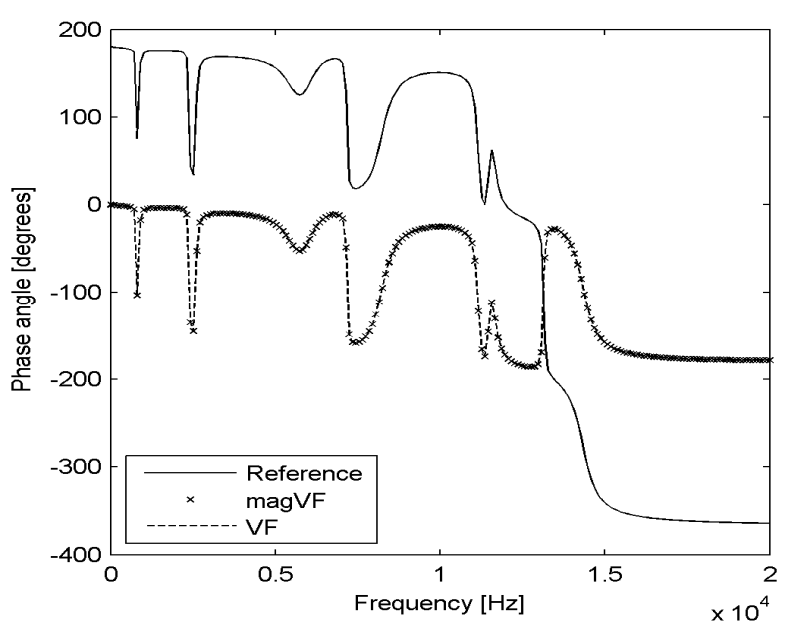

Fig. 2 Phase angle of the reference function $G$ (solid line) compared to magVF approximation (dashed line) and VF approximation (cross).

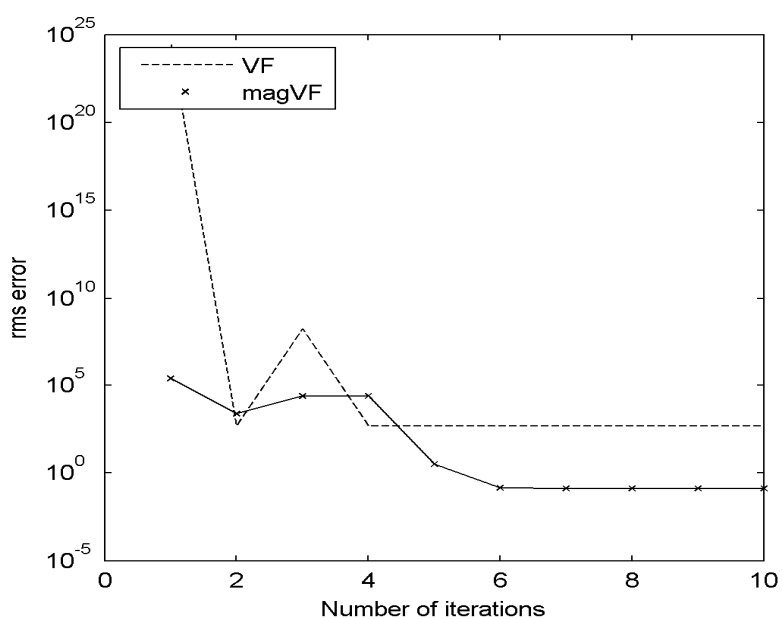

Fig. 4 Comparison between rms-error (13) given by magVF (cross) and VF (dashed line) as function of the number of iterations. 


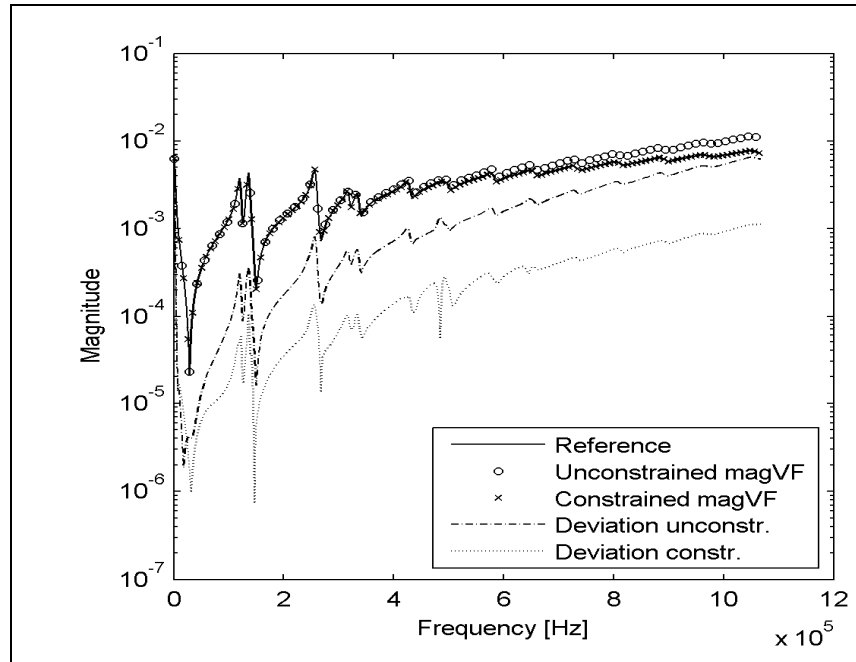

Fig. 5 Magnitude of the reference function $\mathrm{y}_{11}$ (solid line) compared to magVF approximation with constrained (cross) and unconstrained (circle) residue identification.

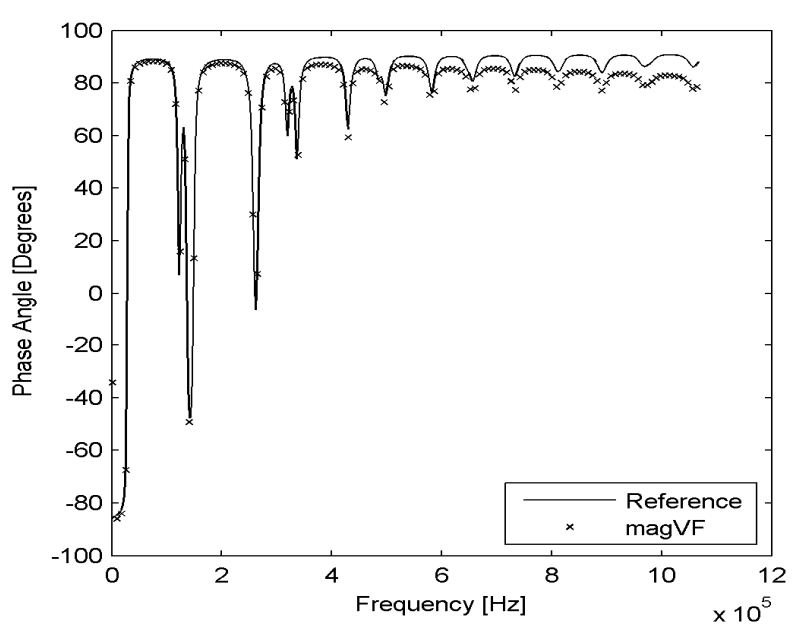

Fig. 6 Phase angle of the reference function $\mathrm{y}_{11}$ (solid) compared to magVF approximation (cross)

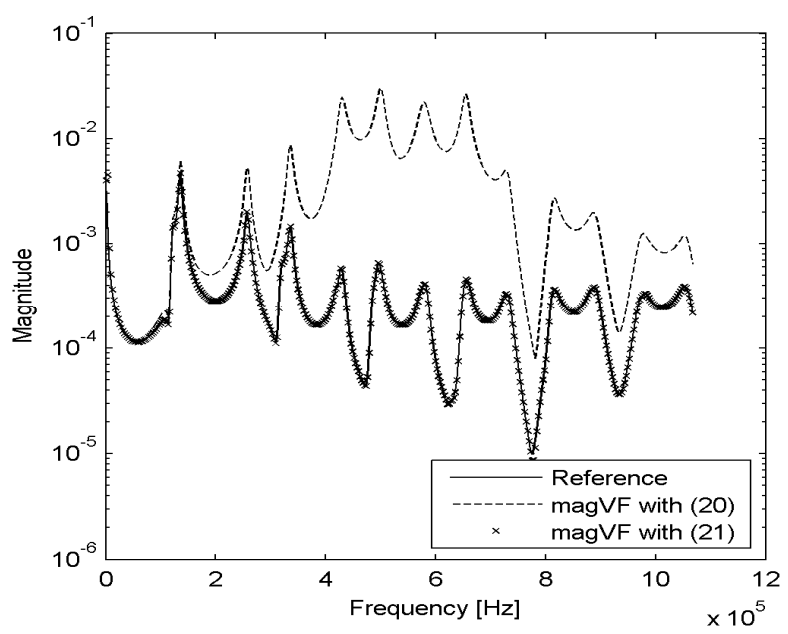

Fig. 7 Magnitude of the reference function $\mathrm{y}_{12}$ (solid) compared to magVF approximations obtained by replacing imaginary poles with either real poles (20) (dashed) or with complex conjugate pairs (21) (cross).

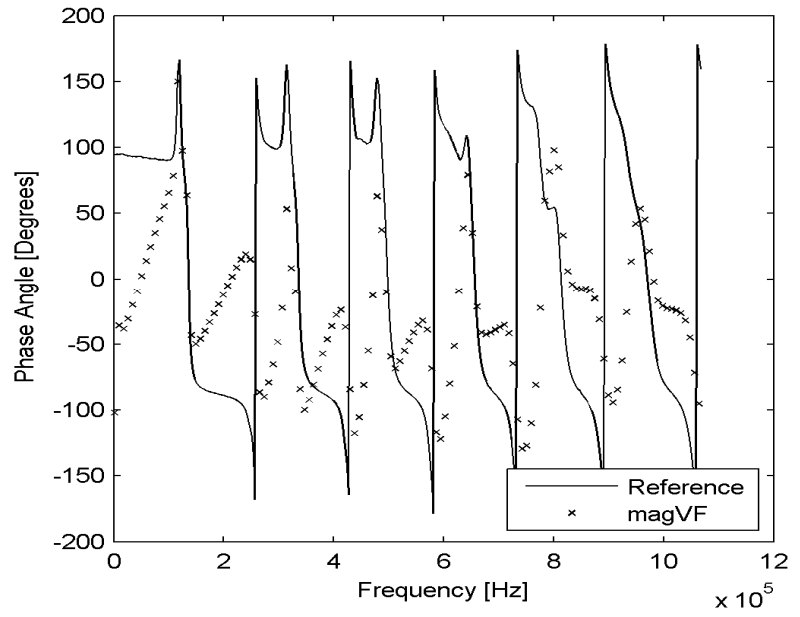

Fig. 8 Phase angle of the reference function $\mathrm{y}_{12}$ (solid) compared to magVF approximation. Transformation of imaginary poles into complex conjugate pairs by (21) (cross). 


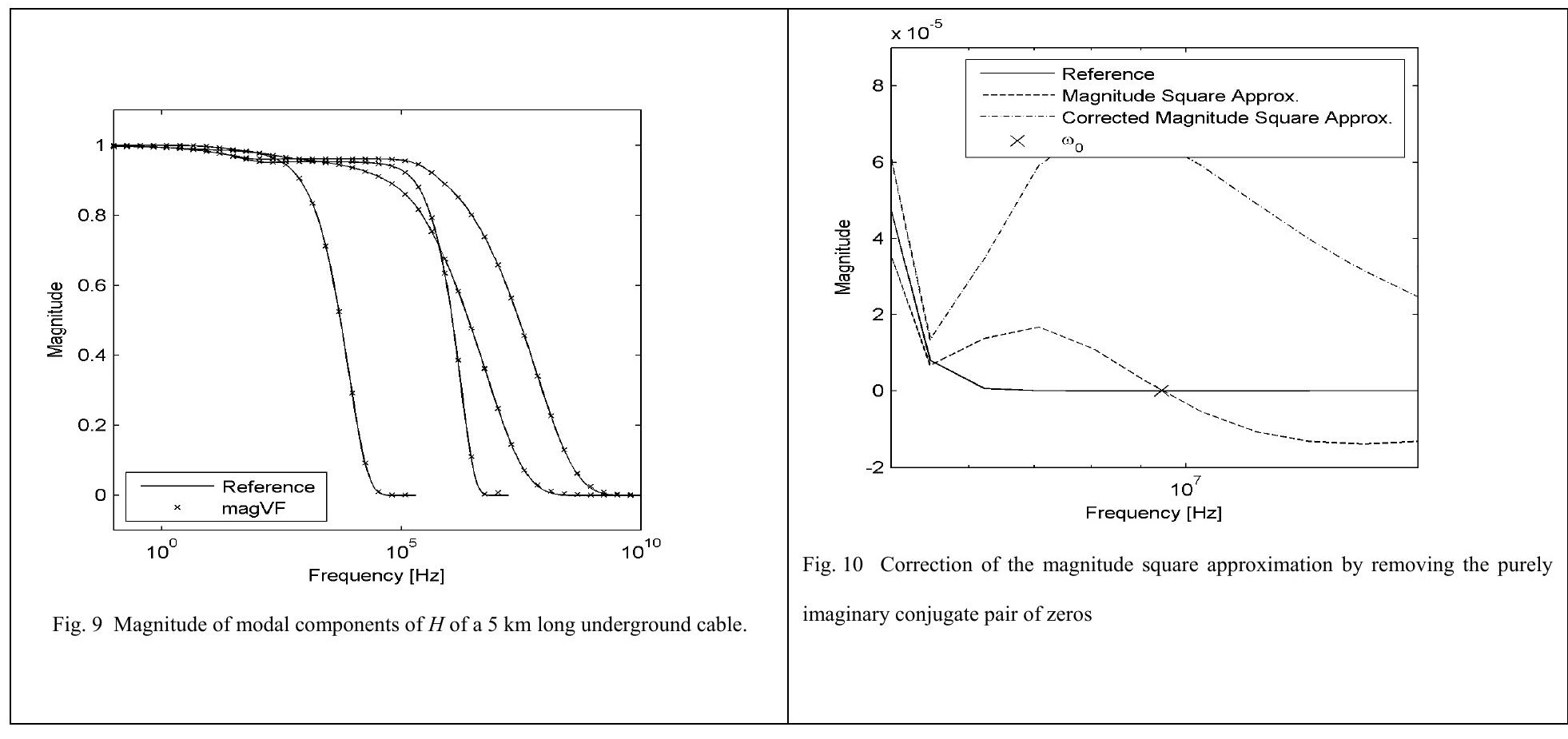

TABLE I

TEST FUNCTION $G(S)$ : POLES AND RESIDUES

\begin{tabular}{|c|c|}
\hline Poles $a_{i}$ & Residues $c_{i}$ \\
\hline-4500 & -3000 \\
\hline-41000 & -83000 \\
\hline$-100 \pm j 5000$ & $-5 \pm j 7000$ \\
\hline$-120 \pm j 15000$ & $-20 \pm j 18000$ \\
\hline$-3000 \pm j 35000$ & $6000 \pm j 45000$ \\
\hline$-20 \odot \pm j 450 \odot \odot$ & $40 \pm j 6 \odot \odot \odot \odot$ \\
\hline$-1500 \pm j 4500 \odot$ & $90 \pm j 1000 \odot$ \\
\hline$-500 \pm j 70000$ & $50000 \pm j 80000$ \\
\hline$-1000 \pm j 7300 \odot$ & $1000 \pm j 45000$ \\
\hline$-2000 \pm j 900 \odot \odot$ & $-500 \odot \pm j 9200 \odot$ \\
\hline
\end{tabular}

